\title{
Immunochromatographic Tests for Mycotoxins Detection with the Use of Ultrasmall Magnetite Nanoparticles ${ }^{\dagger}$
}

\author{
Alina V. Petrakova, Alexandr E. Urusov, Anatoly V. Zherdev * and Boris B. Dzantiev \\ A.N. Bach Institute of Biochemistry, Research Centre of Biotechnology of the Russian Academy of Sciences, \\ 119071 Moscow, Russia; alina.petrakova@gmail.com (A.V.P.); urusov.alexandr@gmail.com (A.E.U.); \\ dzantiev@inbi.ras.ru (B.B.D.) \\ * Correspondence: zherdev@inbi.ras.ru \\ + Presented at the 7th Electronic Conference on Sensors and Applications, 15-30 November 2020; \\ Available online: https://ecsa-7.sciforum.net/.
}

Published: 2 April 2021

\begin{abstract}
The use of small (with a diameter of 7-12 nm) superparamagnetic $\mathrm{Fe}_{3} \mathrm{O}_{4}$ nanoparticles as carriers for antibodies in lateral flow immunoassay is considered. Increased total surface area for such suspension provides a concentration of analytes with an increase in their concentration up to 50 times. When the concentrated complexes were redissolved, aggregates with diameters of 100-500 nm were obtained, serving as colored markers in the assay. Further, magnetite-antibodies complexes are more tolerant to methanol (up to $30 \%$ ) than native antibodies, thus providing minimal dilution of tested extracts. Lateral flow tests for mycotoxins zearalenone, T2-toxin, and aflatoxin B1 were developed and demonstrated applicability to control food products and raw materials.
\end{abstract}

Keywords: immunochromatography; iron oxide; nanoparticles; immobilized antibodies; precipitates; colored labels; mycotoxins; food safety

\section{Introduction}

Dispersions of magnetic substances are popular carriers for antibodies used for immunoanalytical purposes [1-4]. Their choice is based on two reasons. First, magnetic carriers can be manipulated easily. The dispersed micro- or nanoparticles can be evenly distributed in a solution. After immune interactions, the application of a magnetic field rapidly and completely separates the particles with bound molecules from the reaction mixture. Thus, homogeneous interactions are integrated with the creation of a heterogeneous liquid-sediment system for further measurements. Second, magnetic particles can be detected in minimal quantities by their physical properties. The application of magnetic particles makes it possible to reduce analysis time, reach lower detection limits, and facilitate testing of complex substances.

Generally, comparatively large (about $100 \mathrm{~nm}$ or more in diameter) multidomain iron oxide $\left(\mathrm{Fe}_{3} \mathrm{O}_{4}\right)$ particles with a surface coating that protects them from aggregation are used for analytical purposes and prevail in rapid immunoassays such as immunochromatographic (lateral flow) tests [48]. However, their application limits the total contact surface for binding the analyte from the volume of the tested sample. Small single-domain magnetite particles are usually excluded from consideration as possible carriers because they lose their magnetization and form aggregates after application and removal of the magnetic field.

The presented study has shown that the use of such aggregates as optical labels in immunochromatography makes it possible to take all benefits of immunomagnetic concentration and directly register the formed labeled complexes without elution of the bound analyte or other additional 
manipulations. Mycotoxins zearalenone, T2-toxin, and aflatoxin B1 were chosen as target analytes due to importance of their control for food safety $[9,10]$.

\section{Materials and Methods}

For the synthesis of magnetic carriers, 30\% ammonia hydrate was added dropwise, reaching a concentration of $2.4 \%$ to an aqueous solution containing $1.4 \mathrm{mg} / \mathrm{ml} \mathrm{FeCl}_{2}$ and $3.6 \mathrm{mg} / \mathrm{ml} \mathrm{FeCl}$. The mixture was incubated for $15 \mathrm{~min}$ at room temperature with vigorous stirring. The particles were separated by fivefold deposition in a magnetic field with resuspension in $50 \mathrm{mM}$ phosphate buffer, $\mathrm{pH}$ 7.4, with $0.1 \mathrm{M} \mathrm{NaCl}$ [11].

For adsorptive immobilization of antibodies, $500 \mu \mathrm{L}$ of the resulting suspension of magnetite particles with a concentration of $3 \mathrm{mg} / \mathrm{mL}$ were mixed with a solution of antibodies having a final concentration of $60 \mu \mathrm{g} / \mathrm{mL}$. The mixture was incubated for $30 \mathrm{~min}$ at room temperature with vigorous stirring. The modified particles were deposited in a magnetic field and washed three times with the phosphate buffer. We used monoclonal antibodies against T2-toxin and aflatoxin B1 manufactured by IL-Test Pushchino, Ltd. (Pushchino, Russia) and monoclonal antibodies against zearalenone from Eximio Biotec Co., Ltd. (Hangzhou, China) for this immobilization. When preparing immunochromatographic test strips, conjugates of bovine serum albumin with T2-toxin (BioTeZ Berlin-Buch GmbH, Berlin, Germany), aflatoxin B1 (IL-Test Pushchino, Ltd.), and zearalenone (Eximio Biotec Co., Ltd.) were immobilized in the analytical zone, and polyclonal goat antibodies against mouse immunoglobulins manufactured by Arista Biologicals (Allentown, PA, USA) were immobilized in the control zone [11,12].

\section{Results and Discussion}

To obtain dispersed magnetite preparations with small nanoparticles, the synthesis conditions were optimized by varying the concentrations of the reducing agent, $\mathrm{Fe}(\mathrm{II})$ and $\mathrm{Fe}(\mathrm{III})$ salts, for the incubation's duration and temperature regime. The size, shape, and heterogeneity of the obtained nanoparticles and their antibody conjugates were characterized by transmission electron microscopy (see Figure 1). The data obtained showed the nanoparticles' preparation consists of small particles that form aggregates and chains. When isolating individual particles on micrographs, it was found that their average diameter is $9.1 \pm 3.2 \mathrm{~nm}$, and the ellipticity coefficient is $1.4 \pm 0.2$. The sizes of the aggregates vary significantly and can reach hundreds of nanometers in length.

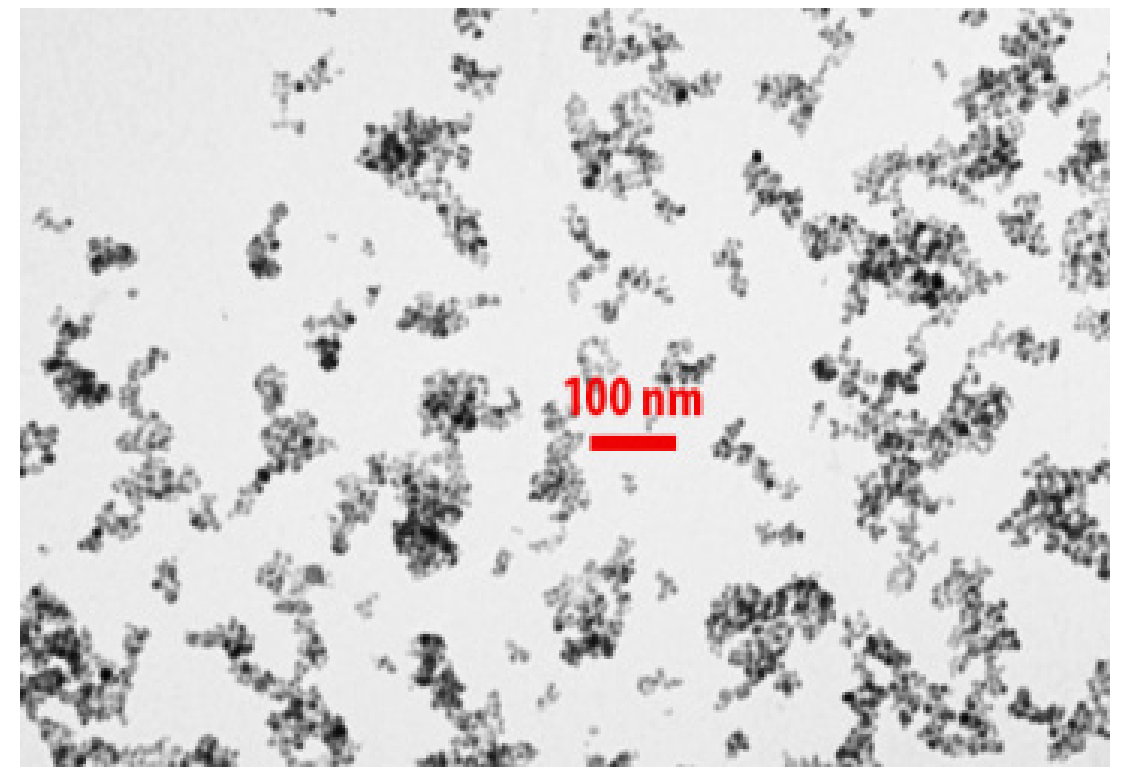

Figure 1. Micrograph of the magnetite nanoparticles conjugating with antibodies. 
The obtained conjugates were first tested by the enzyme-linked immunosorbent assay (ELISA) technique, and the immobilized antibodies' ability to bind target mycotoxins in different reaction media was characterized. It was found that the bound antibodies were tolerant to a higher content of organic solvents (up to $30 \%$ methanol) than native antibodies (significant inactivation occurred even in $15 \%$ methanol). The extraction of mycotoxins from the tested samples by water/organic mixtures is a common stage of sample preparation to further immunoassay. Moreover, the antibody tolerance to organic extractants is an essential parameter determining the necessary dilution of the extract before its contact with antibodies and its loss in the assay sensitivity caused by this dilution. The common practice of the work with soluble antibodies indicates the affinity of antibodies begins to decrease when the presence of methanol or ethanol in the reaction medium becomes higher than 10-15\% (depending on the properties of the studied antibodies). For the most typical extraction medium, $70 \%(\mathrm{v} / \mathrm{v})$ methanol and $30 \%(\mathrm{v} / \mathrm{v})$ water, this means a fivefold dilution of samples before the testing [10]. The increased stability of antibodies in their complexes with magnetite nanoparticles allows this dilution to be reduced to twofold.

Using the obtained conjugates, immunochromatographic tests for detecting mycotoxins zearalenone, T2-toxin, and aflatoxin B1 were developed. The applicability of superparamagnetic nanocarriers as tools to concentrate the target analytes was studied. It was demonstrated that the magnetic separation provides quantitative concentration of the analyte: From $5 \mathrm{~mL}$ of the initial sample to $0.1 \mathrm{~mL}$ of the resuspended magnetic nanoparticles, with its complete binding ensured in 10-15 min. In this case, the nanoparticle-antibody-antigen complexes can be applied directly to the test strip without elution of the antigen. The obtained aggregated nanoparticles with diameters of 100-500 nm can be detected as intense orange-colored markers.

The immunochromatography conditions were determined by eliminating nonspecific binding of these aggregates to membranes. A specific decision on the design of the test strip involved the exclusion of the conjugate pad (because the conjugate is already in the reaction mixture with the sample) and the starting absorbent membrane (it hinders the movement of nanoparticle aggregates, whereas the need to bind the matrix components before they enter the working membrane is eliminated due to the magnetic separation of the matrix). The overall scheme of the proposed assay is presented in Figure 2.

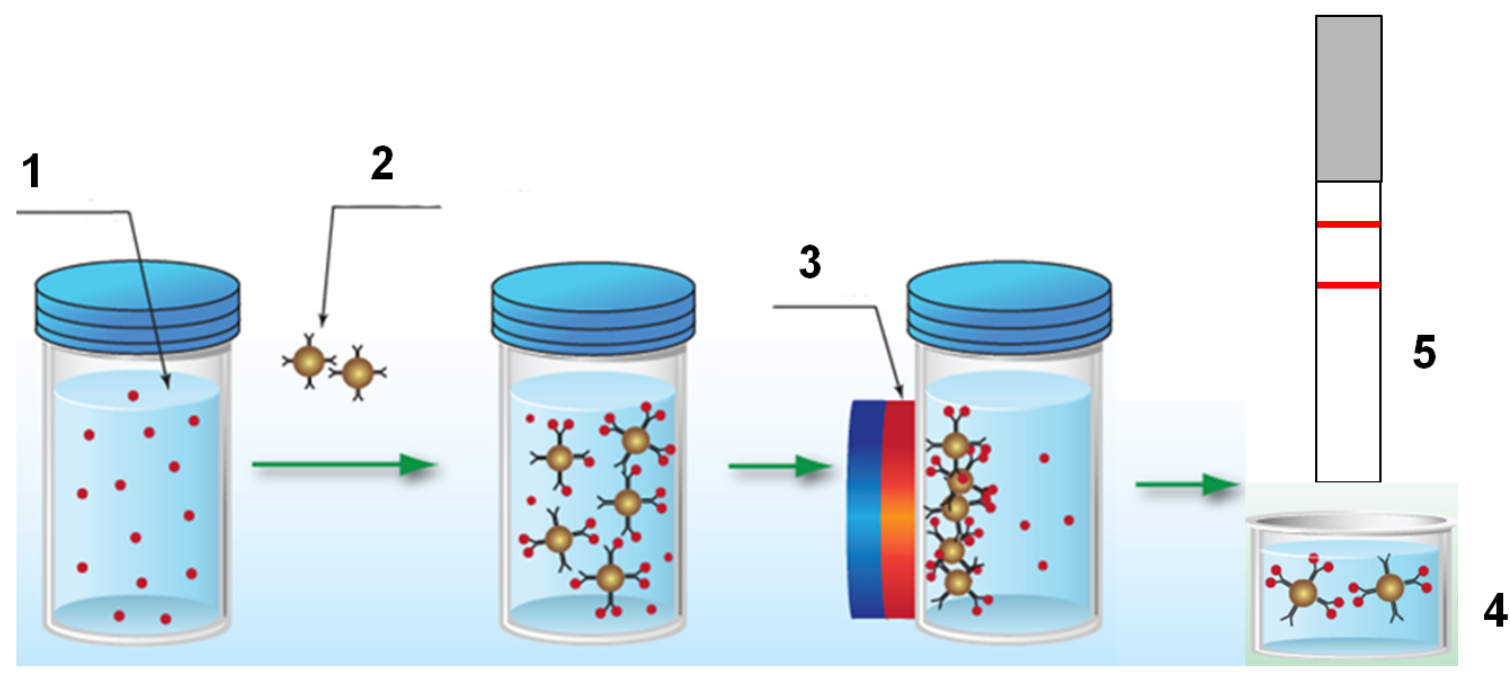

Figure 2. Scheme of immunochromatographic analysis with concentration of the conjugate of magnetic nanoparticles. 1 -tested sample, 2-conjugate of magnetite nanoparticles with antibodies, 3-magnet used to separate the formed immune complexes, 4-sediment dissolved in a small volume, 5-test strip formed from the working membrane (with analytical and control binding zones) and final absorbent pad. 
To account for the need to ensure efficient movement of large aggregates along the membrane, candidate detergents were screened as components of the working buffer. The considered variants included phosphate buffer with the following additives:

- $1 \%$ Tween-20,

- $1 \% \mathrm{BSA}+1 \%$ Tween,

- $1 \%$ sucrose $+1 \%$ Tween-20,

- $0.1 \%$ SDS, and

- $1 \%$ Triton X-100.

The completeness of the elution of the absorbed marker, the uniformity of its movement with the liquid front, and the highest degree of binding in the analytical zone with low nonspecific staining of the membrane was observed for $1 \%$ Tween-20, which was chosen as the optimal detergent. Additional testing of the effect of Tween-20 concentration showed the analysis parameters did not practically change at the level of detergent from $0.5 \%$ and higher, whereas lower levels of detergent led to slowdown of the fluid movement and uneven distribution of the marker along the membrane during immunochromatography.

The selected conditions ensured a high reproducibility of binding with good visual and instrumental detection of colored areas. The average deviation of the color intensity did not exceed 10\% (see Figure 3).

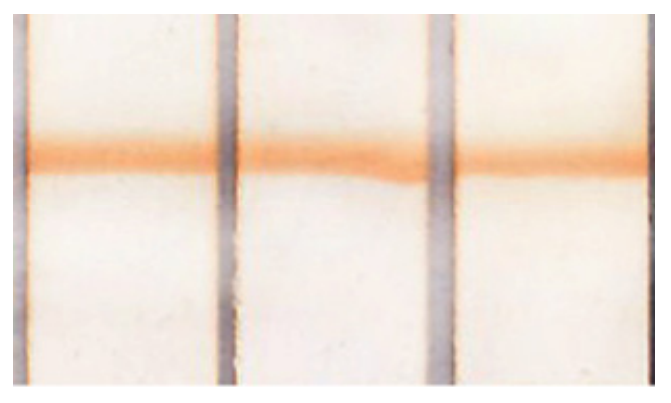

Figure 3. Reproducibility of the colored analytical zones after immunochromatographic assay of T-2 toxin for three samples with concentration of $0.3 \mathrm{ng} / \mathrm{mL}$.

To characterize the proposed assays, we used unpurified extracts ( $70 \%$ methanol, $30 \%$ water) of corn, wheat, and oatmeal grains that did not contain mycotoxins according to preliminary chromatographic testing and were contaminated with known amounts of mycotoxins. The detection limits for mycotoxins reached $0.6-1.3 \mathrm{ng} / \mathrm{mL}$ for visual and $0.05-0.1 \mathrm{ng} / \mathrm{mL}$ for instrument detection. The analysis time was $15 \mathrm{~min}$. These results indicated the effectiveness of the created tests as a means to detect mycotoxin contamination of food products and raw materials, in accordance with high standards for child nutrition.

\section{Conclusions}

The presented data confirm that ultrasmall superparamagnetic nanoparticles integrate stabilization of immobilized antibodies, efficient separation of target analyte molecules from complex matrices, and optical labeling for immunochromatographic assays. These advantages and the simple, low-cost means of acquisition determine such nanoparticles' competitive potential as components of immunosensoric systems for a variety of practically significant analytes.

Author Contributions: Conceptualization, B.B.D.; methodology, A.E.U. and A.V.Z.; validation, A.V.P. and A.E.U.; formal analysis, A.E.U. and A.V.Z.; investigation, A.V.P.; resources, A.E.U. and A.V.Z.; data curation, A.E.U.; writing-original draft preparation, A.V.Z.; writing-review and editing, A.V.P., A.E.U. and B.B.D.; visualization, A.V.P. and A.V.Z.; supervision, A.E.U.; project administration, A.V.Z.; funding acquisition, B.B.D. All authors have read and agreed to the published version of the manuscript.

Funding: The Russian Science Foundation funded this research, grant number 19-14-00370. 
Conflicts of Interest: The authors declare no conflict of interest.

\section{References}

1. Schrittwieser, S.; Pelaz, B.; Parak, W.J.; Lentijo-Mozo, S.; Soulantica, K.; Dieckhoff, J.; Ludwig, F.; Guenther, A.; Tschope, A.; Schotter, J. Homogeneous biosensing based on magnetic particle labels. Sensors 2016, 16, 828, doi:10.3390/s16060828.

2. Urusov, A.E.; Petrakova, A.V.; Zherdev, A.V.; Dzantiev, B.B. Application of magnetic nanoparticles in immunoassay. Nanotechnol. Russia 2017, 12, 471-479, doi:10.1134/S19950780170501.

3. Ha, Y.; Ko, S.; Kim, I.; Huang, Y.M.; Mohanty, K.; Huh, C.; Maynard, J.A. Recent advances incorporating superparamagnetic nanoparticles into immunoassays. ACS Appl. Nano Mater. 2018, 1, 512-521, doi:10.1021/acsanm.7b00025.

4. Moyano, A.; Serrano-Pertierra, E.; Salvador, M.; Martinez-Garcia, J.C.; Rivas, M.; Blanco-Lopez, M.C. Magnetic lateral flow immunoassays. Diagnostics 2020, 10, 288, doi:10.3390/diagnostics10050288.

5. Denmark, D.J.; Bustos-Perez, X.; Swain, A.; Phan, M.H.; Mohapatra, S.; Mohapatra, S.S. Readiness of magnetic nanobiosensors for point-of-care commercialization. J. Electron. Mater. 2019, 48, 4749-4761, doi:10.1007/s11664-019-07275-7.

6. Cai, Y.Y.; Yan, J.; Zhu, L.; Wang, H.L.; Lu, Y. A rapid immunochromatographic method based on a secondary antibody-labelled magnetic nanoprobe for the detection of hepatitis B preS2 surface antigen. Biosensors 2020, 10, 161, doi:10.3390/bios10110161.

7. Cao, B.; Wang, K.; Xu, H.; Qin, Q.; Yang, J.C.; Zheng, W.; Jin, Q.H.; Cui, D.X. Development of magnetic sensor technologies for point-of-care testing: Fundamentals, methodologies and applications. Sens. Actuators A Phys. 2020, 312, 112130, doi:10.1016/j.sna.2020.112130.

8. Zhang, T.; Lei, L.L.; Tian, M.L.; Ren, J.J.; Lu, Z.S.; Liu, Y.; Liu, Y.S. Multifunctional Fe3O4@Au supraparticle as a promising thermal contrast for an ultrasensitive lateral flow immunoassay. Talanta 2021, 222, 121478, doi:10.1016/j.talanta.2020.121478.

9. Rahman, H.U.; Yue, X.F.; Yu, Q.Y.; Xie, H.L.; Zhang, W.; Zhang, Q.; Li, P.W. Specific antigen-based and emerging detection technologies of mycotoxins. J. Sci. Food Agric. 2019, 99, 4869-4877, doi:10.1002/jsfa.9686.

10. Tittlemier, S.A.; Cramer, B.; Dall'Asta, C.; Iha, M.H.; Lattanzio, V.M.T.; Maragos, C.; Solfrizzo, M.; Stranska, M.; Stroka, J.; Sumarah, M. Developments in mycotoxin analysis: An update for 2018-19. World Mycotoxin J. 2020, 13, 3-24, doi:10.3920/WMJ2019.2535.

11. Petrakova, A.V.; Urusov, A.E.; Zherdev, A.V.; Liu, L.; Xu, C.; Dzantiev, B.B. Application of magnetite nanoparticles for the development of highly sensitive immunochromatographic test systems for mycotoxin detection. Appl. Biochem. Microbiol. 2017, 53, 470-475, doi:10.1134/S0003683817040111.

12. Urusov, A.E.; Petrakova, A.V.; Zherdev, A.V., Dzantiev, B.B. Magnetic nanopartices as carriers for immunoassays. Nano Hybrids Compos. 2017, 13, 54-62, doi:10.4028/www.scientific.net/nhc.13.54.

Publisher's Note: MDPI stays neutral with regard to jurisdictional claims in published maps and institutional affiliations.

(C) 2021 by the authors. Licensee MDPI, Basel, Switzerland. This article is an open access article distributed under the terms and conditions of the Creative Commons Attribution (CC BY) license (http://creativecommons.org/licenses/by/4.0/). 